\title{
Habitable Zone Super-Earths with Non-Stabilised Spectrographs
}

\author{
Duncan J. Wright, Christopher G. Tinney and \\ Robert A. Wittenmyer \\ University of New South Wales, \\ Sydney, NSW 2052, Australia \\ email: duncan.wright@unsw.edu.au
}

\begin{abstract}
Detecting the small velocity amplitudes $(\leqslant 10 \mathrm{~m} / \mathrm{s})$ produced by habitable zone rocky planets around M Dwarfs requires radial velocity precisions of a few $\mathrm{m} \mathrm{s}^{-1}$. However, an iodine absorption cell, commonly used as a high precision wavelength reference on non-stabilised spectrographs, is not efficient for very red and faint objects like M Dwarfs. Instead, arc lamps have to be used. With the exception of the ultra-stabilised HARPS spectrograph, achieving $\sim \mathrm{m} \mathrm{s}^{-1}$ calibration with arc lamps has not been possible because typical spectrographs experience drifts of several hundred $\mathrm{m} \mathrm{s}^{-1}$ due to local atmospheric changes in pressure and temperature. We outline and present results from an innovative differential wavelength calibration method that enables $\sim \mathrm{m} \mathrm{s}^{-1}$ precision from non-stabilised, high-resolution spectrographs. This technique allows the detection of rocky planets with radial velocity amplitudes of a few $\mathrm{ms}^{-1}$.
\end{abstract}

Keywords. instrumentation: spectrographs, methods: data analysis, techniques: spectroscopic, techniques: radial velocities

\section{Introduction}

Finding Earth-like planets orbiting in the habitable zone of other stars is one of the major goals of modern astronomy. Detecting Earth-like planets, in Earth-like orbits around Sun-like stars is currently beyond reach of any facility except Kepler (and is problematic even then). What is possible now, is the detection of Earth-like planets in habitable-zone orbits around low-mass stars using the radial velocity technique. Early indications from both Doppler planet searches and Kepler are that rocky planets around low-mass stars $\left(<1 \mathrm{M}_{\odot}\right)$ are common $(>30 \%$, see e.g. Wesley Traub's, Jessie Christiansen's and Courtney Dressing's contributions to this volume). Several M Dwarf planet searches have begun in recent years [e.g., HARPS - Bonfils et al. (2013); ROPS - Barnes et al. (2012); CRIRES - Bean et al. (2010); NIRSPEC - Bailey et al. (2012)] looking in the optical through to the IR. We are initiating a search, sensitive to low-mass planets orbiting in the habitable zones of M4-M6 dwarf stars. This search will utilize a new high-precision arc-lamp wavelength calibration method developed for the CYCLOPS fibre-feed to the UCLES spectrograph on the AAT and will operate over $\sim 500-950 \mathrm{~nm}$. Table 1 demonstrates why M-dwarfs make such compelling targets for habitable zone searches. A $5 \mathrm{M}_{\oplus}$ planet will orbit a $0.3 \mathrm{M}_{\odot}$ star in periods of just $4.5-70 \mathrm{~d}$. For an even smaller $0.1 \mathrm{M}_{\odot}$ star they orbit in just 3.5-18d. These short orbital periods make such planets far more detectable. A $5 \mathrm{M}_{\oplus}$ planet orbiting a M-dwarf with mass between $0.1-0.3 \mathrm{M}_{\odot}$ delivers Doppler amplitudes of $1.8-9.8 \mathrm{~m} \mathrm{~s}^{-1}$. 
Table 1. Orbital Properties of a $5 \mathrm{M}_{\oplus}$ Habitable Zone Planet Orbiting an M Dwarf

\begin{tabular}{|c|c|c|c|c|c|c|c|}
\hline \multicolumn{2}{|c|}{ Host M Dwarf } & \multicolumn{2}{|c|}{ Semi-major Axis (AU) } & \multicolumn{2}{|c|}{ Period (d) } & \multicolumn{2}{|c|}{ Doppler Amplitude $\left(\mathrm{m} \mathrm{s}^{-1}\right)$} \\
\hline | Mass $\left(\mathrm{M}_{\odot}\right)$ & Sp. Type & | Inner & Outer & | Inner & Outer & Inner & Outer \\
\hline 0.1 & M6 & 0.021 & 0.061 & $3.5 \mathrm{~d}$ & $18 \mathrm{~d}$ & 9.8 & 5.7 \\
\hline 0.3 & M4 & 0.09 & 0.21 & $4.5 \mathrm{~d}$ & $70 \mathrm{~d}$ & 4.4 & 1.8 \\
\hline
\end{tabular}

\section{The calibration process}

This new differential calibration method uses a single reference wavelength solution determined from a normal Thorium-Argon (ThAr) exposure which is hereafter termed the reference ThAr. All subsequent ThAr calibration images are used to measure how the position of the spectrum has changed. The changes are then fitted and applied as a modification to the reference ThAr's wavelength solution.

There are several benefits to such an approach. The wavelength solution of the reference image is computed similarly to a traditional method, and therefore has similar errors. However, the differential technique produces more consistent solutions for all subsequent ThArs than the traditional independently computed ones. The benefits of this technique come from the fact that we can measure changes to the ThAr spectrum position with fewer parameters than we need to describe a complete new position. Additionally, the cross-correlation technique used to determine the shift along an extracted order can be made to include many weak lines. These lines are stable but are normally too weak to use in the traditional isolated line fitting approach. This results in improved precision of the difference measurement between a ThAr and the reference ThAr image.

Shifts in a ThAr spectrum are measured by a fit to the cross-correlation of chunks of it's extracted spectrum with the reference ThAr. The shifts from different chunks are mapped to the corresponding part of the $2 \mathrm{D}$ image via the order trace, where they form a continuous surface. A low-order 2D polynomial is fitted to this surface which is then mapped into the absolute order number $(\mathrm{m})$ versus extracted pixel ( $\mathrm{x}$ ) space using the order trace. In the $m$ vs. $x$ space this surface fit describes the pixel shifts to be applied to the reference ThAr's wavelength solution.

\section{An example: CYCLOPS on the AAT}

CYCLOPS is a single object integral field unit made up of 15 close-packed 0.6 " hexagonal fibres (only 12 functioning) that are arranged to have a $\sim 2.5$ " diameter on the sky. The fibres are reformatted to make a pseudo slit that injects light into UCLES at resolution $\sim 70000$. Each of the 12 fibres produces its own spectrum and can be extracted, calibrated and used for RV measurements separately. An upgrade to the CYCLOPS fibre feed is being commissioned at present. The upgrade is a similar design but with two extra on-sky fibres (for a total of 17 sky fibres) and a simultaneous arc lamp calibration fibre.

The UCLES spectrograph is not temperature or pressure stabilised. It is an open air spectrograph with several moving parts. At the end of each observing run optical components such as the echelle grating are parked and covered. It has been used for highprecision Doppler planet work using an Iodine cell, which accounts for zero point and point-spread-function changes in the spectrograph independently in many small chunks of the stellar+Iodine spectrum. The Iodine cell method is not useful for M Dwarfs so we have been developing reduction and calibration methods to optimise UCLES+CYCLOPS RV precision using arc lamps. The spectrograph experiences shifts up to half a pixel $\left(\sim 800 \mathrm{~m} \mathrm{~s}^{-1}\right)$ in both the echelle-dispersion and cross-dispersion directions during a 
typical night. The main causes of these shifts are the camera liquid Nitrogen cooling cycle and atmospheric pressure changes.

Following the procedure outlined in the above section and using 400 pixel chunk sizes for our cross-correlations, preliminary results at $\sim 400-700 \mathrm{~nm}$ have obtained $5 \mathrm{~m} \mathrm{~s}^{-1}$ rootmean-squared precisions for Tau Ceti over 5 months.

\section{Working in the infra-red}

The M Dwarf stars that will be observed are nearby, but are intrinsically faint. Most M dwarfs are $\mathrm{V}>9$ mag. They emit most of their flux in the infra-red and tend to be $\sim 2$ magnitudes brighter in the I band. The M Dwarfs are cool, and so have a molecular absorption forest in the infra-red which can provide a large amount of RV information, hence we have decided to focus our survey on the infra-red. To use the calibration technique outlined above in the infra-red requires a calibration lamp that has as many emission features in the optical-to-infra-red as possible. We are currently investigating Thorium-Argon, Thorium-Xenon and Uranium-Neon lamps for this calibration. So far our investigation has shown that the Thorium-Xenon lamp offers fewer super-saturating noble-gas lines in the infra-red region than the Uranium-Neon and Thorium-Argon lamps. Despite the bright Neon lines, Uranium-Neon lamps have been shown to have many more useable infra-red lines than Thorium-Argon lamps (Redman et al., 2011) and are therefore expected to be the most suitable calibration lamp.

\section{Conclusions}

Utilizing the new differential arc lamp calibration method discussed here, and the CYCLOPS integral field unit looking in the infra-red, we expect to achieve $\sim 5 \mathrm{~ms}^{-1}$ precisions on a selection of M4-M6 candidates. With sufficient epochs these data will be precise enough to detect habitable zone super Earths around the M Dwarf sample and will be second only to HARPS in precision for current M Dwarf studies.

\section{References}

Bonfils, X., Delfosse, X., Udry, S., Forveille, T., Mayor, M., Perrier, C., Bouchy, F., Gillon, M., Lovis, C., Pepe, F., Queloz, D., Santos, N. C., Sgransan, D., \& Bertaux, J.-L. 2013, 549, A109

Barnes, J. R., Jenkins, J. S., Jones, H. R. A., Rojo, P., Arriagada, P., Jordn, A., Minniti, D., Tuomi, M., Jeffers, S. V., \& Pinfield, D. 2012, MNRAS V. 424, pp. 591-604

Bean, Jacob L., Seifahrt, Andreas, Hartman, Henrik, Nilsson, Hampus, Wiedemann, Gnter, Reiners, Ansgar, Dreizler, Stefan, Henry, \& Todd J. 2010, ApJ V. 713, pp. 410-422

Bailey, John I., III, White, Russel J., Blake, Cullen H., Charbonneau, Dave Barman, Travis S., Tanner, Angelle M., \& Torres, Guillermo 2012, ApJ V. 749, art. 16

Redman, Stephen L., Lawler, James E., Nave, Gillian, Ramsey, Lawrence W., \& Mahadevan, Suvrath 2011, ApJS V. 195, art. 24 